\title{
The clinical and histological characteristics of patients with biopsy-proven non-alcoholic fatty liver disease in the absence of insulin resistance
}

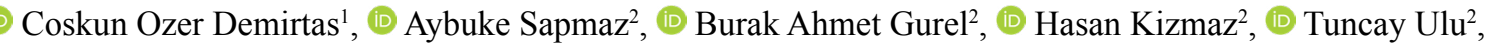 \\ (D) Seyma Gorcin Karaketir ${ }^{3}$, (D) Seyhan Hidiroglu ${ }^{3}$, (D) Yusuf Yilmaz ${ }^{1,4}$ \\ ${ }^{I}$ Department of Gastroenterology, Marmara University School of Medicine, Istanbul, Turkey; ${ }^{2}$ Marmara University School of Medicine, Istanbul, Turkey; \\ ${ }^{3}$ Department of Public Health, Marmara University School of Medicine, Istanbul, Turkey; ${ }^{4}$ Marmara University Institute of Gastroenterology, \\ Istanbul, Turkey
}

\begin{abstract}
Background and Aim: Non-alcoholic fatty liver disease (NAFLD) is closely associated with insulin resistance (IR). While a minority of patients with NAFLD does not have evidence of IR, no detailed characterization of this specific phenotype is currently available. This study was undertaken to investigate the clinical and histological characteristics of this patient group.

Materials and Methods: We retrospectively reviewed the clinical records of 263 patients with biopsy-proven NAFLD. IR was defined by a Homeostatic Model Assessment of IR (HOMA-IR) score $>2.73$. The histological diagnosis of non-alcoholic steatohepatitis (NASH) was performed using 1) the steatosis, activity and fibrosis score and 2) the NASH Clinical Research Network (NASH CRN) criteria. Significant fibrosis was defined by the presence of a histological fibrosis score higher than $\mathrm{F} \geq 2$. Patients with and without evidence of IR were compared concerning clinical, laboratory, and histological characteristics.
\end{abstract}

Results: Of the 263 patients with biopsy-proven NAFLD, 53 (20.2\%) patients had no evidence of IR. Patients without IR were younger [IR (-): $42(22-65)$ years versus IR $(+): 49(22-71)$ years, $p=0.001]$ and had a higher prevalence of men [IR (-): 39 (73.6\%) versus IR (+): $113(53.8 \%)$, $\mathrm{p}=0.009]$. Moreover, they were characterized by a lower body mass index [IR (-): $30.06 \pm 3.61 \mathrm{~kg} / \mathrm{m}^{2}$ versus IR (+): $33.19 \pm 5.06 \mathrm{~kg} / \mathrm{m}^{2}, \mathrm{p}=0.000$ ] and lower frequencies of metabolic risk factors-including the metabolic syndrome, diabetes, hypertension, hyperlipidemia, and lower waist/hip circumference. Liver histology was generally less severe in patients without IR; specifically, they showed a lower prevalence of NASH [IR (-): $38(71.7 \%)$ versus IR $(+): 190(90.5 \%), p=0.000]$ and significant fibrosis [IR (-): $9(17.0 \%)$ versus IR (+): $106(50.5 \%), \mathrm{p}=0.000]$ than did patients

How to cite this article: Demirtas CO, Sapmaz A, Gurel BA, Kizmaz H, Ulu T, Gorcin Karaketir S, et al. A disquiet find: The clinical and histological characteristics of patients with biopsy-proven non-alcoholic fatty liver disease in the absence of insulin resistance. Hepatology Forum 2020; 1(3):101-108.

Received: July 06, 2020; Accepted: July 09, 2020; Available online: September 21,2020

Corresponding author: Coskun Ozer Demirtas; Marmara Universitesi Tip Fakultesi, Gastroenteroloji Anabilim Dali, Istanbul, Turkey

Phone: +90 50591761 12; e-mail: coskun_demirtas10@hotmail.com

(c) (1) OPEN ACCESS

(1) This work is licensed under a Creative Commons Attribution-NonCommercial 4.0 International License.

๑ Copyright 2020 by Hepatology Forum - Available online at www.hepatologyforum.org with IR. Multivariate analysis identified obesity [odds ratio (OR): 9.321, 95\% confidence interval $(\mathrm{CI}): 1.031-84.261, \mathrm{p}<0.05]$ and an international normalized ratio $>1.1$ (OR: 10.116, 95\% CI: $1.325-77.225$, $\mathrm{p}<0.05$ ) as independent predictors of significant fibrosis in patients with NAFLD and no IR.

Conclusion: Patients with NAFLD and no IR has less severe liver histology than patients with IR. However, obesity appears to be independently associated with significant fibrosis in this patient group.

Keywords: Fibrosis; histology; HOMA score; insulin resistance; non-alcoholic fatty liver disease.

\section{Introduction}

Non-alcoholic fatty liver disease (NAFLD) is characterized by the presence of excessive hepatic fat accumulation ( $>5 \%)$ in the absence of other potential causes of steatosis. NAFLD encompasses a wide spectrum from simple steatosis to steatohepatitis (NASH), and subsequently to cirrhosis and hepatocellular carcinoma. ${ }^{[1,2]}$ NAFLD has long been considered as the hepatic component of metabolic syndrome, and it is strongly correlated with its manifestations such as obesity and type 2 diabetes mellitus. ${ }^{[3,4]}$ In parallel to the growing obesity epidemic, the prevalence of type 2 diabetes mellitus, metabolic syndrome and NAFLD has increased over the last two decades. ${ }^{[5]}$ Recent meta-analysis showed that the overall prevalence of NAFLD among patients with type 2 diabetes mellitus is $55.5 \%$, whereas the global prevalence of NASH among patients with type 2 diabetes mellitus is $37.3 \%{ }^{[6]}$ On the other hand, several epidemiological studies have revealed a closer association of NAFLD with insulin resistance (IR) rather than metabolic syndrome itself and its clinical manifestations. ${ }^{[7,8]}$ Therefore, NAFLD is, in fact, currently considered as the hepatic manifestation of IR.

Traditionally, the existence of IR has been mentioned as the first hit of a two-hit hypothesis constructed to explain the pathogenesis of NASH. [9] Accordingly, IR led to simple steatosis as the first hit, whereas the second hit was done by oxidative stress, lipid peroxidation and mitochondrial dysfunction. However, in recent years, there is an increasing recognition that NAFLD is a way more heterogeneous disease with multiple pathways of pathogenesis. ${ }^{[10]}$ The pivotal role of IR in the development and progression of NAFLD has been demonstrated 
multiple times and is well-acknowledged. However, the pathogenesis of NAFLD without underlying IR remains dubious and a little is known about the characteristics of this relatively rare subgroup.

To date, the issue of whether NAFLD without IR is a different entity than the NAFLD with IR is still unclarified. Therefore, we sought to expose the characteristics of NAFLD arising in the absence of IR by comparing it with a group of patients with NAFLD with underlying IR. Moreover, we aimed to identify the independent predictors of significant fibrosis $(F \geq 2)$ in patients with NAFLD without IR.

\section{Materials and Methods}

\section{Study Population and Design}

The present study was designed as a retrospective review of prospectively collected data. In this study, 515 consecutive adult patients with biopsy-proven NAFLD recruited from the outpatient facilities of the department of gastroenterology at a tertiary center were investigated. Indications for liver biopsy were as follows: evidence of hepatic steatosis on ultrasound, abnormal liver enzymes or hepatomegaly, and absence of secondary causes of hepatic fat accumulation [e.g., significant alcohol consumption ( $>21$ units of alcohol per week for men and $>14$ units of alcohol per week for women) and previous history of steatogenic drugs use]. Patients were excluded from this study if they met one of the following criteria; the presence of viral hepatitis, drug-induced liver disease, autoimmune hepatitis, and genetic liver diseases. A complete medical history was taken, and all underwent a comprehensive physical examination before the biopsy procedure. Initial demographic, laboratory and clinical parameters were recorded before the liver biopsy procedure. Aspartate transaminase value was considered normal if lower than $37 \mathrm{U} / \mathrm{L}$ using the upper limits of our laboratory, while upper limits of normal alanine transaminase were determined as $32 \mathrm{U} / \mathrm{L}$ for men and $23 \mathrm{U} / \mathrm{L}$ for women in accordance with the recently published data in a Turkish population. ${ }^{[11,12]}$ Obesity is defined for patients with a body-mass index (BMI) value higher than $30 \mathrm{~kg} / \mathrm{m}^{2}$, whereas overweight is described for patients with a BMI value between 25 and $29.99 \mathrm{~kg} / \mathrm{m}^{2}$. Patients who are not overweight or obese are categorized as having lean BMI.

\section{Identification of Groups with and without Insulin Resistance}

IR was defined using the most commonly and globally used proxy in clinical practice, namely The Homeostatic Model Assessment of IR (HOMA-IR) score. The threshold for identifying IR was accepted as 2.73 , as it is the most widespread acknowledged cut-off value for the distinction. ${ }^{[1]}$ HOMA-IR score was calculated as follows: fasting glucose $(\mathrm{mmoL} / \mathrm{L}) \mathrm{x}$ fasting insulin $(1 \mathrm{U} / \mathrm{mL}) / 22.5$. Patients with HOMA score $\geq 2.73$ were classified in IR $(+)$ group, whereas patients with HOMA score $<2.73$ were grouped as IR (-). To limit the influence of supra-physiological results of insulinemia induced by insulin therapy, patients with insulin use were excluded from both groups. Besides, patients using anti-diabetic drugs were also excluded from the low HOMA group, as it may have reduced the inherent HOMA score of subjects. After the exclusion of ineligible cases, a total of 263 patients were enrolled for the analysis. The flow diagram of the study is exhibited in Figure 1.

\section{Liver Histology}

Liver biopsies were processed by an experienced pathologist. A

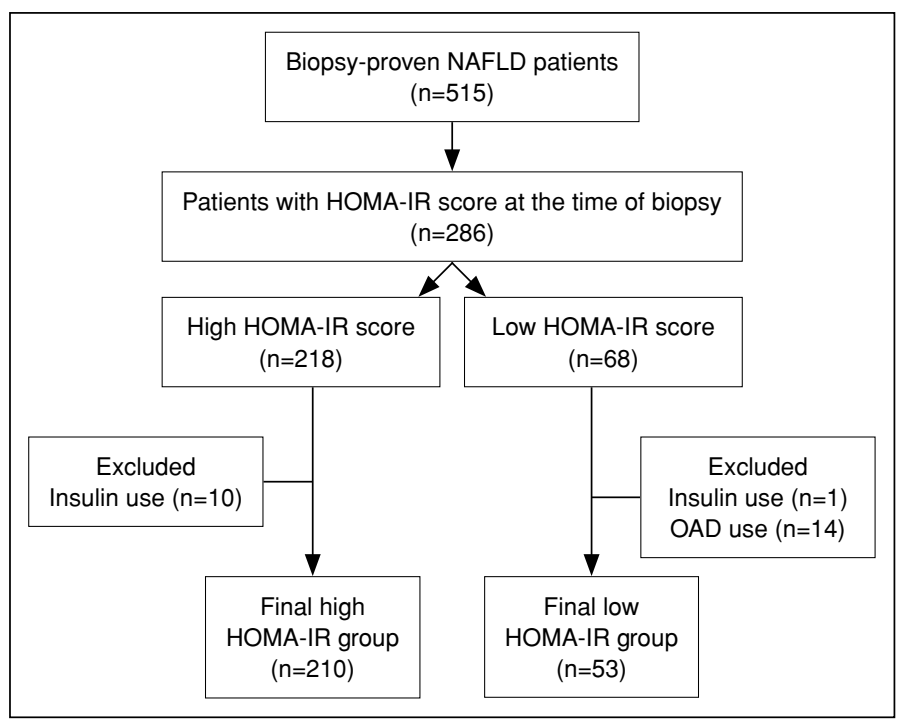

Figure 1. Flow diagram of this study.

biopsy length of at least $1.5 \mathrm{~cm}$ or the presence of six to eight portal tracts are considered adequate and was also excluded if inadequate. ${ }^{[12]}$ An expert pathologist classified all liver biopsy specimens as nonNASH versus NASH using the steatosis, activity and fibrosis (SAF) scoring system in accordance with the fatty liver inhibition of progression algorithm. ${ }^{[13]} \mathrm{NASH}$ was diagnosed when all three histological parameters, steatosis, hepatocellular ballooning and lobular inflammation, had a grade of 1 or more. NAFLD activity score (NAS) was calculated based on the sum of the following three parameters: steatosis (ranging from 0 to 3 ), hepatocellular ballooning (ranging from 0 to 2). NAS scores were categorized as follows; $<3$ : nonNASH, 3-4: borderline NASH, $>4$ : definite NASH. NASH Clinical Research Network criteria were applied for each specimen according to NAS score as published. ${ }^{[14]}$ The NAFLD severity was considered mild when the activity grade was $<2$ and the fibrosis stage was $<2$, whereas severe disease was assigned when both were $\geq 2 .{ }^{[15]}$ Significant fibrosis was defined as a histological fibrosis score of higher than $F \geq 2$, whereas advanced fibrosis was defined as a histological fibrosis score of higher than $\mathrm{F} \geq 3 .{ }^{[16]}$

\section{Statistical Analysis}

Data were presented using descriptive statistics. Intergroup comparisons of normally distributed and skewed continuous variables were performed using the Student's t-test and the Mann-Whitney U test, respectively. Pearson's chi-square test was used to analyse categorical variables. Multivariate logistic regression analyses were performed using the significantly different parameters in definitive univariate analysis to reveal the independent predictors of significant fibrosis in both groups. The statistical significance was defined as $\mathrm{p}<0.05$. All statistical analyses were conducted using the SPSS software version 20.0 (IBM, Armonk, NY, USA).

\section{Ethics}

This study followed the tenets of the Helsinki Declaration, and it was approved by the local Ethics Committee (Protocol No: 09.2019.808). Owing to the retrospective nature of this study, the need for informed consent was waived. 
Table 1. Demographics of the study population

\begin{tabular}{|c|c|c|c|c|c|c|c|}
\hline & \multicolumn{2}{|c|}{$\begin{array}{c}\text { IR (+) } \\
(n=210)\end{array}$} & \multicolumn{2}{|c|}{$\begin{array}{l}\operatorname{IR}(-) \\
(n=53)\end{array}$} & \multicolumn{2}{|c|}{$\begin{array}{l}\text { Whole cohort } \\
\quad(n=263)\end{array}$} & \multirow[t]{2}{*}{$\mathbf{p}$} \\
\hline & $\mathbf{n}$ & $\%$ & $\mathbf{n}$ & $\%$ & $\mathbf{n}$ & $\%$ & \\
\hline Age, median (IQR), y & \multicolumn{2}{|c|}{$49(22-71)$} & \multicolumn{2}{|c|}{$42(22-65)$} & \multicolumn{2}{|c|}{$47(22-71)$} & $0.001^{*}$ \\
\hline \multicolumn{8}{|l|}{ Sex } \\
\hline Male & 113 & 53.8 & 39 & 73.6 & 152 & 57.8 & $0.009^{*}$ \\
\hline \multicolumn{8}{|l|}{ Cigarette } \\
\hline Active & 30 & 15.3 & 11 & 22 & 41 & 16.7 & \\
\hline Quit & 51 & 26 & 11 & 22 & 62 & 25.2 & 0.503 \\
\hline Never smoked & 115 & 58.7 & 28 & 56 & 143 & 58.1 & \\
\hline \multicolumn{8}{|l|}{ Alcohol } \\
\hline $\mathrm{BMI}$, mean $\pm \mathrm{SD}, \mathrm{kg} / \mathrm{m}^{2}$ & \multicolumn{2}{|c|}{$33.19 \pm 5.06$} & \multicolumn{2}{|c|}{$30.06 \pm 3.61$} & \multicolumn{2}{|c|}{$31.71 \pm 5.0$} & $0.000^{*}$ \\
\hline \multicolumn{8}{|l|}{ BMI category } \\
\hline Lean & 11 & 5.2 & 4 & 7.5 & 15 & 5.7 & \\
\hline Overweight & 51 & 24.3 & 24 & 47.2 & 76 & 28.9 & $0.002^{*}$ \\
\hline Obese & 148 & 70.5 & 24 & 45.3 & 172 & 65.4 & \\
\hline Waist circumference, mean $\pm S D, c m$ & \multicolumn{2}{|c|}{$107.4 \pm 11.3$} & \multicolumn{2}{|c|}{$101.1 \pm 8.2$} & \multicolumn{2}{|c|}{$105.9 \pm 10.9$} & $0.001^{*}$ \\
\hline Hip circumference, median (IQR), cm & \multicolumn{2}{|c|}{$110.9(63-155)$} & \multicolumn{2}{|c|}{$107(94-129)$} & \multicolumn{2}{|c|}{$109(63-155)$} & $0.013^{*}$ \\
\hline Type 2 diabetes mellitus & 102 & 48.6 & 7 & 6.4 & 109 & 41.4 & $0.000^{*}$ \\
\hline Hypertension & 89 & 42.4 & 11 & 20.8 & 100 & 38 & $0.004^{*}$ \\
\hline Hyperlipidemia & 115 & 54.8 & 17 & 32.1 & 132 & 50.2 & $0.003^{*}$ \\
\hline
\end{tabular}

IQR: Interquartile range; BMI: Body-mass index; IR: Insulin resistance; SD: Standard deviation; *: Sign indicates significant $p$-values as $<0.05$.

\section{Results}

\section{General Characteristics of Patients with NAFLD with or without Underlying IR}

Demographics of the study population are summarized in Table 1. Of the 263 eligible patients with biopsy-proven NAFLD, 53 (20.2\%) patients had no concomitant IR. Compared with IR (+) group, patients in IR (-) group were younger [IR (-): 42 (22-65) years vs. IR (+): 49 (22-71) years, $\mathrm{p}=0.001$ ], and more commonly male [IR (-): 39 (73.6\%) vs. IR (+): 113 $(53.8 \%), \mathrm{p}=0.009]$. Moreover, patients in IR (-) group had lower BMI [IR (-): $30.06 \pm 3.61 \mathrm{~kg} / \mathrm{m}^{2}$ vs. IR (+): $\left.33.19 \pm 5.06 \mathrm{~kg} / \mathrm{m}^{2}, \mathrm{p}=0.000\right]$ and less frequently associated metabolic risk factors (metabolic syndrome, diabetes, hypertension, hyperlipidaemia, and lower waist/hip circumference).

In the laboratory, albumin, creatinine and low-density lipoprotein (LDL) were higher, and thyroid-stimulating hormone (TSH), INR, HbAlc and spot microalbuminuria was lower in the IR (-) group compared to IR (+) group. C-peptide, a cleavage product of insulin synthesis that is co-secreted with insulin, was also lower in the IR (-) group in line with the lower insulin levels. Laboratory characteristics of the study population are presented in Table 2.

\section{Histopathological Characteristics of Patients with NAFLD with or without Underlying IR}

The histopathological characteristics of the study population are pre- sented in Table 3. IR (-) group differed from IR (+) group concerning the following histological findings: grade of steatosis (S), stage of activity (A), stage of fibrosis (F), NAS score, as well as ballooning, lobular inflammation and portal inflammation. All aforementioned findings increased in the IR $(+)$ group in comparison with IR (-) group. The diagnosis of NASH was observed more frequently in the IR $(+)$ group according to both SAF algorithm classification [IR (-): 38 (71.7\%) vs. IR (+): $190(90.5 \%), p=0.000]$ and NASH CRN criteria. The liver histology in the IR $(+)$ group tended to be more severe, with higher rates of severe disease than the IR (-) group. Both significant fibrosis [IR (-): $9(17.0 \%)$ vs. IR $(+): 106(50.5 \%), \mathrm{p}=0.000]$ and advanced fibrosis [IR (-): $9(9.4 \%)$ vs. IR (+): $54(25.7 \%), \mathrm{p}=0.011]$ were more common in IR $(+)$ group. Twelve $(5.7 \%)$ patients were diagnosed with NASH-related cirrhosis in the IR (+) group, whereas none of the subjects in the IR (-) group had cirrhosis.

\section{Independent Predictors of Significant Fibrosis in Patients with NAFLD with or without IR}

In multivariate analysis, presence of diabetes mellitus [odds ratio $(\mathrm{OR})=2.096,95 \%$ confidence interval $(\mathrm{CI})=1.012-4.341, \mathrm{p}=0.046]$, hypertension $(\mathrm{OR}=3.501,95 \% \mathrm{CI}=1.601-7.658)$, older age $(\mathrm{OR}=1.037$, 95\% CI=1.001-1.074), GGT $(\mathrm{OR}=2.618,95 \% \mathrm{CI}=1.286-5.332)$, platelet count $[<200 \times 1000 / \mathrm{m} 3](\mathrm{OR}=3.329,95 \% \mathrm{CI}=1.286-5.332)$, 
Table 2. Laboratory characteristics of the study population

\begin{tabular}{|c|c|c|c|c|}
\hline & $\begin{array}{l}\text { IR (+) } \\
(n=210)\end{array}$ & $\begin{array}{l}\text { IR }(-) \\
(n=53)\end{array}$ & $\begin{array}{l}\text { Whole cohort } \\
(n=263)\end{array}$ & $\mathbf{p}$ \\
\hline Normal transaminase, $\mathrm{n}(\%)$ & $51(24.3)$ & $14(26.4)$ & $65(24.7)$ & 0.725 \\
\hline AST, U/L & $41(11-168)$ & $35(16-149)$ & $40(11-168)$ & 0.112 \\
\hline ALT, U/L & $63(12-288)$ & $59(18-215)$ & $62(12-288)$ & 0.306 \\
\hline ALP, U/L & $82(34-563)$ & $90(38-178)$ & $83(34-563)$ & 0.661 \\
\hline GGT, U/L & $48(9-559)$ & $49(13-217)$ & $48(9-559)$ & 0.567 \\
\hline Creatinine, mg/dL & $0.81 \pm 0.2$ & $0.92 \pm 0.16$ & $0.83(0.41-2.13)$ & $0.000^{*}$ \\
\hline $\mathrm{LDH}, \mathrm{U} / \mathrm{L}$ & 207 (19-449) & $203(150-328)$ & $205(19-449)$ & 0.130 \\
\hline Total bilirubin, $\mathrm{mg} / \mathrm{dL}$ & $0.71(0.1-6.1)$ & $0.75(0.28-2.8)$ & $0.72(0.1-6.1)$ & 0.399 \\
\hline Direct bilirubin, mg/dL & $0.14(0.1-1.83)$ & $0.15(0.1-0.88)$ & $0.14(0.1-1.83)$ & 0.853 \\
\hline Total protein, gr/dL & $7.7(6.4-9.14)$ & $7.8(6.5-8.5)$ & $7.7(6.4-9.1)$ & 0.109 \\
\hline Albumin, gr/dL & $4.5(3.5-5.9)$ & $4.7(3.9-5.6)$ & $4.6(3.5-5.9)$ & $0.001^{*}$ \\
\hline Total cholesterol, mg/dL & $206(74-419)$ & $211(93-417)$ & $208(74-419)$ & 0.563 \\
\hline LDL, mg/dL & $129(28-400)$ & $145(31.2-259)$ & $133(28-400)$ & $0.03^{*}$ \\
\hline $\mathrm{HDL}, \mathrm{mg} / \mathrm{dL}$ & $44(19-78)$ & $43(25-74)$ & $44(19-78)$ & 0.704 \\
\hline Triglycerides, mg/dL & $163(37-716)$ & $169(44-1107)$ & $164(37-1107)$ & 0.399 \\
\hline $\mathrm{TSH}, \mathrm{mIU} / \mathrm{L}$ & $1.59(0.1-9.52)$ & $1.14(0.23-22.1)$ & $1.52(0.1-13.3)$ & $0.005^{\star}$ \\
\hline Uric acid, mg/dL & $6.25 \pm 1.52$ & $6.02 \pm 1.04$ & $6.21 \pm 1.43$ & 0.339 \\
\hline White blood cell count, $\mu \mathrm{L} / \mathrm{mL}$ & $6.980(2.400-14.900)$ & $6.500(4.800-12.300)$ & $6.900(2.400-14.900)$ & 0.601 \\
\hline Hemoglobin, $g / L$ & $14.5(8.5-18.9)$ & $14.8(7.1-17)$ & $14.6(7.1-18.9)$ & 0.180 \\
\hline Platelet count, $\times 1000 / \mathrm{m}^{3}$ & $229(77-421)$ & $243(89-543)$ & $232(77-543)$ & 0.252 \\
\hline INR & $1.02(0.87-1.7)$ & $0.98(0.85-1.2)$ & $1.01(0.8-1.9)$ & $0.033^{*}$ \\
\hline Ferritin, ng/mL & $92.7(4.1-645)$ & $90(6.65-395.2)$ & $92(4.1-645)$ & 0.99 \\
\hline $\mathrm{TIBC}, \mathrm{ug} / \mathrm{dL}$ & $368.5 \pm 71.1$ & $364.8 \pm 58.6$ & $367.7 \pm 68.6$ & 0.556 \\
\hline Glucose, mg/dL & $103(77-307)$ & $89(66-117)$ & $100(66-307)$ & $0.000^{*}$ \\
\hline Insulin, $\mu \mathrm{IU} / \mathrm{mL}$ & $17.9(9.13-76.12)$ & $8.8(1.85-13)$ & $16.4(1.85-76.12)$ & $0.000^{*}$ \\
\hline HOMA-IR & $4.71(2.73-28.76)$ & $2.02(0.32-2.6)$ & $4.07(0.32-28.76)$ & $0.000^{*}$ \\
\hline $\mathrm{HbA} 1 \mathrm{c}$ & $5.9(4-11.1)$ & $5.5(4.6-6.2)$ & $5.8(4-11.1)$ & $0.000^{*}$ \\
\hline C-peptid, ng/mL & $3.69(1.08-261)$ & $2.68(1.74-10.2)$ & $3.5(1.08-261)$ & $0.003^{*}$ \\
\hline Spot microalbuminuria, $\mathrm{mg} / \mathrm{L}$ & $13.65(1-2925.01)$ & $5.12(0.1-641.9)$ & $11.51(0.1-2925.01)$ & $0.000^{*}$ \\
\hline
\end{tabular}

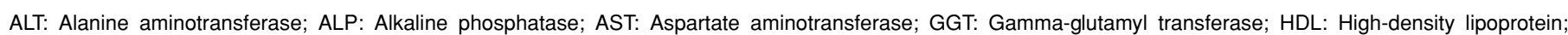

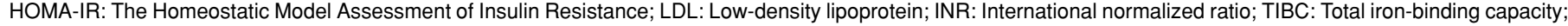
*: Sign indicates significant $p$-values as $<0.05$. Data were given as mean \pm standard deviation or median (interquartile range) when appropriate.

higher INR $[>1.1](\mathrm{OR}=2.533,95 \% \mathrm{CI}=1.251-5.132, \mathrm{p}=2.533)$ and increasing HOMA score $(\mathrm{OR}=1.101,95 \% \mathrm{CI}=1.001 .211, \mathrm{p}=0.049)$ were identified as independent predictors of significant fibrosis in IR ( + ) group (Table 4). In IR (-) group, obesity (OR=9.321, 95\% CI=1.03184.261) and having higher INR [ $>1.1](\mathrm{OR}=10.116,95 \% \mathrm{CI}=1.325-$ $77.225, \mathrm{p}=0.026$ ) were the only independent predictors of significant fibrosis (Table 5).

\section{Discussion}

To our knowledge, this is the first study assessing the characteristics and revealing the specific predictors of significant fibrosis in patients with all biopsy-proven NAFLD without concomitant IR. Our study revealed that one in every five patients with NAFLD does not have underlying IR, and cases in this subgroup are younger, thinner (lower BMI, waist and hip circumference) and more commonly male with less metabolic comorbidities. The rate of NAFLD without IR in our study was lower than previous reports from Asia, giving a prevalence of $46 \%$ to $65.9 \%$.
${ }^{[17-19]}$ This discrepancy can be explained by that the majority of their patients were not considered candidates for liver biopsy and diagnosed with ultrasonography only. Our cohort consisted of patients solitarily biopsy-proven NAFLD, of whom more than $85 \%$ was NASH. Therefore, our results are more representative of NASH rather than NAFLD, which may explain the higher rates of underlying IR in our patients.

To date, several reports have investigated the potential association of IR with the severity of liver histology. In a previous study from India conducted in 285 patients with biopsy-proven NAFLD; steatosis, NAS score and risk of having NASH increased in NAFLD patients with IR compared to patients without IR, but they could not demonstrate any difference in other parameters, including the stage of fibrosis. ${ }^{[20]}$ In our study, the findings showed that patients with NAFLD without IR possess less severe liver histology concerning nearly all histologic parameters, as well as fibrosis stage. In 2014, Jung et al. ${ }^{[18]}$ reported that HOMA-IR values were higher in subjects with NASH than healthy controls, but could not demonstrate a significant correlation of 
Table 3. Histological characteristics of the study population

\begin{tabular}{|c|c|c|c|c|c|c|c|}
\hline \multirow{3}{*}{ Length of specimen, $\mathrm{mm}$} & \multicolumn{2}{|c|}{$\begin{array}{c}\text { IR (+) } \\
(n=210)\end{array}$} & \multicolumn{2}{|c|}{$\begin{array}{c}\operatorname{IR}(-) \\
(n=53)\end{array}$} & \multicolumn{2}{|c|}{$\begin{array}{l}\text { Whole cohort } \\
\qquad(n=263)\end{array}$} & \multirow[t]{2}{*}{$\mathbf{p}$} \\
\hline & $\mathbf{n}$ & $\%$ & $\mathbf{n}$ & $\%$ & $\mathbf{n}$ & $\%$ & \\
\hline & \multicolumn{2}{|c|}{$29(7-65)$} & \multicolumn{2}{|c|}{$28(17-49)$} & \multicolumn{2}{|c|}{$29(7-65)$} & 0.956 \\
\hline Number of portal tracts, median (IQR) & \multicolumn{2}{|c|}{$20(6-41)$} & \multicolumn{2}{|c|}{$19(11-20)$} & \multicolumn{2}{|c|}{$20(6-41)$} & 1.000 \\
\hline \multicolumn{8}{|l|}{ Grade of steatosis (S) } \\
\hline S2 & 89 & 42.4 & 21 & 39.6 & 110 & 41.8 & 0.001 \\
\hline S3 & 88 & 41.9 & 12 & 22.6 & 100 & 38 & \\
\hline \multicolumn{8}{|l|}{ Grade of activity $(\mathrm{A})$} \\
\hline A0 & 1 & 0.5 & 3 & 5.7 & 4 & 1.5 & \\
\hline $\mathrm{A} 1$ & 15 & 7.1 & 12 & 22.6 & 27 & 10.3 & \\
\hline \multicolumn{8}{|l|}{ Stage of fibrosis (F) } \\
\hline Fo & 30 & 14.3 & 27 & 50.9 & 57 & 21.7 & \\
\hline $\mathrm{F} 1$ & 74 & 35.2 & 17 & 32.1 & 91 & 34.6 & \\
\hline F2 & 52 & 24.8 & 4 & 7.5 & 56 & 21.3 & 0.000 \\
\hline F3 & 42 & 20 & 5 & 9.4 & 47 & 17.9 & \\
\hline $\mathrm{F} 4$ & 12 & 5.7 & - & - & 12 & 4.6 & \\
\hline \multicolumn{8}{|l|}{ SAF algorithm classification } \\
\hline NAFL & 20 & 9.5 & 15 & 28.3 & 35 & 13.3 & 0.000 \\
\hline NASH & 190 & 90.5 & 38 & 71.7 & 228 & 86.7 & \\
\hline Mild/Severe disease & \multicolumn{2}{|c|}{$16(7.6) / 194(92.4)$} & \multicolumn{2}{|c|}{$14(26.4) / 39(73.6)$} & \multicolumn{2}{|c|}{$30(11.4) / 233(88.6)$} & 0.000 \\
\hline 1 & 107 & 51 & 34 & 64.2 & 141 & 53.6 & 0.002 \\
\hline 2 & 92 & 43.8 & 11 & 20.8 & 103 & 39.2 & \\
\hline \multicolumn{8}{|l|}{ Portal inflammation } \\
\hline 0 & 56 & 31.1 & 35 & 70 & 91 & 39.6 & \\
\hline 1 & 97 & 53.9 & 15 & 30 & 112 & 48.7 & 0.000 \\
\hline 2 & 25 & 13.9 & - & - & 25 & 10.9 & \\
\hline 3 & 2 & 1.1 & - & - & 2 & 0.9 & \\
\hline NAS score, median (IQR) & 4 & $1-8$ & 4 & $1-8$ & 5 & $1-8$ & 0.000 \\
\hline \multicolumn{8}{|l|}{ NASH CRN criteria } \\
\hline Non-NASH & 7 & 3.3 & 8 & 15.1 & 15 & 5.7 & \\
\hline Borderline NASH & 55 & 26.2 & 23 & 43.4 & 78 & 29.7 & 0.000 \\
\hline Definite NASH & 148 & 70.5 & 22 & 41.5 & 170 & 64.6 & \\
\hline Significant fibrosis $(\geq \mathrm{F} 2)$ & 106 & 50.5 & 9 & 17.0 & 115 & 43.7 & \\
\hline Advanced fibrosis ( $\geq \mathrm{F} 3$ ) & 54 & 25.7 & 5 & 9.4 & 59 & 22.4 & 0.011 \\
\hline Cirrhosis (F4) & 12 & 5.7 & - & - & 12 & 4.6 & 0.075 \\
\hline
\end{tabular}

CRN: Clinical research network; NAFL: Non-alcoholic fatty liver; NASH: Non-alcoholic steatohepatitis; SAF: Steatosis, activity and fibrosis; *: Sign indicates significant p-values as $<0.05$. 
Table 4. Predictors of significant fibrosis in IR (+) subjects

\begin{tabular}{llllr}
\hline & Univariate & & \multicolumn{1}{c}{ Multivariate } \\
\cline { 2 - 4 } & p value & p value & Exp (B) & 95\% Cl \\
\hline Diabetes & 0.000 & $0.046^{*}$ & 2.096 & $1.012-4.341$ \\
Hypertension & 0.000 & $0.002^{*}$ & 3.501 & $1.601-7.658$ \\
Hyperlipidemia & 0.002 & 0.688 & 1.163 & 1.037 \\
Age & 0.000 & $0.042^{*}$ & 2.618 & $1.001-1.074$ \\
GGT $(>48$ vs. $\leq 48)$ & 0.003 & $0.008^{*}$ & 3.329 & $1.286-5.332$ \\
Platelet count $(<200$ vs $\geq 200)$ & 0.001 & $0.003^{*}$ & 2.533 & $1.520-7.290$ \\
INR $(>1.1$ vs. $\leq 1.1)$ & 0.009 & $0.01^{*}$ & 0.615 & $1.251-5.132$ \\
Creatinine $(>0.9$ vs. $\leq 0.9)$ & 0.000 & 0.298 & $0.247-1.534$ \\
HOMA-IR score & 0.001 & $0.049^{*}$ & 1.101 & $1.000-1.211$ \\
\hline
\end{tabular}

GGT: Gamma-glutamyl transferase; HOMA-IR: The Homeostatic Model Assessment of Insulin Resistance; INR: International normalized ratio; CI: Confidence interval; *: Sign indicates significant $p$-values as $<0.05$.

Table 5. Predictors of significant fibrosis in IR (-) subjects

\begin{tabular}{lllll}
\hline & Univariate & & \multicolumn{2}{c}{ Multivariate } \\
\cline { 2 - 4 } & p value & p value & Exp (B) & 95\% Cl \\
\hline Female gender & 0.03 & 0.346 & 2.917 & $0.315-26.977$ \\
Obese & 0.05 & $0.047^{*}$ & 9.321 & $1.031-84.261$ \\
INR $(>1.1$ vs. $\leq 1.1)$ & 0.046 & $0.026^{*}$ & 10.116 & $1.325-77.225$ \\
Creatinine $(>0.9$ vs. $\leq 0.9)$ & 0.015 & 0.197 & 0.216 & $0.021-2.219$ \\
\hline
\end{tabular}

INR: International normalized ratio; Cl: Confidence interval; *: Sign indicates significant $p$-values as $<0.05$.

HOMA-IR with the severity of histologic stage in 41 patients with biopsy-proven NAFLD, probably due to small sample size. ${ }^{[18]}$ One year later, a multicentre study conducted in Japan with 1365 patients with biopsy-proven NAFLD showed that HOMA-IR significantly increases in line with the increasing degree of steatosis, lobular inflammation, ballooning, fibrosis and NAS score. ${ }^{[20]}$ Another study from Italy with 118 consecutive patients with biopsy-proven NAFLD reported HOMA-IR as an independent predictor of advanced fibrosis. ${ }^{[21]}$ Considering the predominance of type 2 diabetes in these studies (approximately $25 \%$ ), a more recent study analysed 361 patients with biopsy-proven NAFLD without diabetes and aimed to investigate whether HOMA-IR can reflect advanced fibrosis in non-diabetic patients as well. ${ }^{[22]}$ They found a threshold of 2.9 for HOMA-IR to be an independent predictor of advanced fibrosis in non-diabetic NAFLD patients. In our study, we used the most widely accepted threshold for HOMA-IR score (2.73) and showed that HOMA-IR is an indicator of significant fibrosis only in NAFLD patients with IR, but not in NAFLD patients without IR.

In general, older age, obesity, the existence of diabetes and hypertension are well-acknowledged predictors of advanced fibrosis in patients with NAFLD and has been demonstrated several times from different regions of the world. ${ }^{[23-29]}$ Likewise, our results, GGT was shown to predict fibrosis progression in a study, but many others have proven the contradictory results. ${ }^{[29]}$ Besides, another study showed that GGT and HOMA-IR were positively correlated with each other in patients with NAFLD supporting the interplay amongst GGT and HOMA-IR in predicting increasing the stage of fibrosis. ${ }^{[30]}$ Not surprisingly, higher
INR and low platelet count was able to predict significant fibrosis in our study, as 12 patients from NAFLD with IR group were diagnosed with cirrhosis after the biopsy. The predictors of significant fibrosis for patients with NAFLD with IR in our study were generally coherent with the previous literature knowledge, as the majority of the former studies were conducted in cohorts with a predominance of underlying IR. In our study, we investigated the predictors of significant fibrosis $(>F 2)$ in patients with NAFLD without IR for the first time and found out that obesity and having higher INR (>1.1) can be the premonitory sign of significant fibrosis and NASH in this subgroup.

Several differences in the laboratory amongst the two groups can be explained by the histological differences. The lower albumin and LDL detected in the NAFLD patients with IR is probably the indicator of higher rates of advanced fibrosis in this group, as they do decrease in line with the altered liver synthesis functions. Same explanation can be attributed to higher INR detected in patients with NAFLD with IR. The higher value of TSH detected in patients with NAFLD with IR may also be explained by the higher NASH and advanced fibrosis in this group. Interestingly, a recent report has demonstrated the higher occurrence rates of subclinical hypothyroidism and low thyroid functions in NASH and advanced fibrosis as well, supporting the TSH differences found amongst two groups in our study. ${ }^{[31]}$ The reduced levels of creatinine in NAFLD patients with IR group may be reasoned owing to the cirrhotic cases, as patients with advanced chronic liver disease have a significantly lower baseline serum creatinine concentration than the general population due to a $50 \%$ decrease in hepatic production of creatinine. ${ }^{[32]}$ 
Finally, lower HbA1c and spot microalbuminuria in NAFLD patients without IR was not surprising, as nearly half of the NAFLD patients with IR had diabetes comorbidity.

There are several limitations of our study that should be considered when interpreting results. First, the study cohort only consisted of Turkish NAFLD subjects from a tertiary centre. Therefore, the results may not be applicable to other ethnicities and the general population. Secondly, the subject selection for liver biopsy can be biased due to a part that refused to undergo liver biopsy. Finally, the implementation of the hyperinsulinemic-euglycemic clamp test, the gold standard test for IR, could have assessed the IR status of each case more accurately than the HOMA-IR score. However, the availability of liver biopsies in all patients, the larger sample size with prospective follow-up, was the main strength of our study. Besides, to our knowledge, this is the first study to reveal the predictors of significant fibrosis in patients with NAFLD according to IR status. Our study lacks investigation of genetic interactions amongst NAFLD and IR. In a recent study, the association of a PNPLA3 variant (PNPLA3 1148M) with normal insulin sensitivity has been demonstrated. ${ }^{[33]}$ Moreover, the presence of IR was shown to amplify the PNPLA3-rs738409-G genetic risk factor for hepatic steatosis. ${ }^{[34]}$ Further studies are warranted to clarify the underlying role of genetics in the advancement of NAFLD in patients without IR.

In conclusion, our study revealed that NAFLD patients without IR tended to have less severe liver histology compared to patients with underlying IR. While predictors of significant fibrosis in NAFLD patients with IR were generally in line with the previously reported parameters in the literature, obesity appears to be independently associated with significant fibrosis NAFLD patients without IR.

Ethics Committee Approval: This study followed the tenets of the Helsinki Declaration, and it was approved by the local Ethics Committee (date: 13.09.2019, number: 09.2019.808).

Peer-review: Externally peer-reviewed.

Author Contributions: COD: writing, statistical analysis and preparing the final version, AS, BAG, HK, TU: Data collection, SGK: Statistical analyses, YY: Conceptualization, design and final review.

Conflict of Interest: The authors have no conflict of interest to declare.

Financial Disclosure: The authors declared that this study has received no financial support.

\section{References}

1. Chalasani N, Younossi Z, Lavine JE, Charlton M, Cusi K, Rinella M, et al. The diagnosis and management of nonalcoholic fatty liver disease: Practice guidance from the American Association for the Study of Liver Diseases. Hepatology 2018;67(1):328-357.

2. Kaya E, Y1lmaz Y. Non-alcoholic fatty liver disease: A growing public health problem in Turkey. Turk J Gastroenterol 2019;30(10):865-871.

3. Kitade H, Chen G, Ni Y, Ota T. Nonalcoholic Fatty Liver Disease and Insulin Resistance: New Insights and Potential New Treatments. Nutrients 2017;9(4):387.

4. Kaya E, Bakir A, Kani HT, Demirtas CO, Keklikkiran C, Yilmaz Y. Simple Noninvasive Scores Are Clinically Useful to Exclude, Not Predict, Advanced Fibrosis: A Study in Turkish Patients with Biopsy-Proven Nonalcoholic Fatty Liver Disease. Gut Liver 2020;14(4):486-491.

5. Yılmaz Y, Kanı HT, Demirtaş CÖ, Kaya E, Sapmaz AF, Qutranji L, et al. Growing burden of nonalcoholic fatty liver disease in Turkey: A single-center experience. Turk J Gastroenterol 2019;30(10):892-898.

6. Younossi ZM, Golabi P, de Avila L, Paik JM, Srishord M, Fukui N, et al. The global epidemiology of NAFLD and NASH in patients with type 2 diabetes: A systematic review and meta-analysis. J Hepatol 2019 Oct;71(4):793-801.

7. Musso G, Gambino R, Bo S, Uberti B, Biroli G, Pagano G, et al. Should nonalcoholic fatty liver disease be included in the definition of metabolic syndrome? A cross-sectional comparison with Adult Treatment Panel III criteria in nonobese nondiabetic subjects. Diabetes Care 2008;31(3):562-568.

8. Shibata M, Kihara Y, Taguchi M, Tashiro M, Otsuki M. Nonalcoholic fatty liver disease is a risk factor for type 2 diabetes in middle-aged Japanese men. Diabetes Care 2007;30(11):2940-2944.

9. Day CP, James OF. Steatohepatitis: a tale of two "hits"? Gastroenterology 1998;114(4):842-845.

10. Younossi ZM, Reyes MJ, Mishra A, Mehta R, Henry L. Systematic review with meta-analysis: non-alcoholic steatohepatitis - a case for personalised treatment based on pathogenic targets. Aliment Pharmacol Ther 2014;39(1):3-14.

11. Sumner AE, Cowie CC. Ethnic differences in the ability of triglyceride levels to identify insulin resistance. Atherosclerosis 2008;196:696-703.

12. Bravo AA, Sheth SG, Chopra S. Liver biopsy. N Engl J Med 2001;344(7):495500 .

13. Bedossa P, Poitou C, Veyrie N, Bouillot JL, Basdevant A, Paradis V, et al. Histopathological algorithm and scoring system for evaluation of liver lesions in morbidly obese patients. Hepatology 2012;56(5):1751-1759.

14. Kleiner DE, Brunt EM, Van Natta M, Behling C, Contos MJ, Cummings OW, et al; Nonalcoholic Steatohepatitis Clinical Research Network. Design and validation of a histological scoring system for nonalcoholic fatty liver disease. Hepatology 2005;41(6):1313-1321.

15. Bedossa P; FLIP Pathology Consortium. Utility and appropriateness of the fatty liver inhibition of progression (FLIP) algorithm and steatosis, activity, and fibrosis (SAF) score in the evaluation of biopsies of nonalcoholic fatty liver disease. Hepatology 2014;60(2):565-575.

16. Bilgin BO, Sunbul M, Kani HT, Demirtas CO, Keklikkiran C, Yilmaz Y. Arterial stiffness is associated independently with liver stiffness in biopsyproven nonalcoholic fatty liver disease: a transient elastography study. Eur J Gastroenterol Hepatol 2020;32(1):54-57.

17. Das K, Das K, Mukherjee PS, Ghosh A, Ghosh S, Mridha AR, Dhibar $\mathrm{T}$, et al. Nonobese population in a developing country has a high prevalence of nonalcoholic fatty liver and significant liver disease. Hepatology 2010;51(5):1593-602.

18. Jung KY, Cho SY, Kim HJ, Kim SB, Song IH. Nonalcoholic steatohepatitis associated with metabolic syndrome: relationship to insulin resistance and liver histology. J Clin Gastroenterol 2014;48(10):883-888.

19. Alam S, Anam K, Islam S, Mustafa G, Mamun AA, Ahmad N. Clinical, anthropometric, biochemical and histological character of nonalcoholic fatty liver disease without Insulin Resistance. J Clin Exp Hepatol 2019;9(2):176181.

20. Kessoku T, Yoneda M, Sumida Y, Eguchi Y, Fujii H, Hyogo H, et al; Japan Study Group of NAFLD. Insulin resistance correlated with the severity of liver histology in Japanese NAFLD patients: a multicenter retrospective study. J Clin Gastroenterol 2015;49(2):169-170.

21. Ballestri S, Nascimbeni F, Romagnoli D, Lonardo A. The independent predictors of non-alcoholic steatohepatitis and its individual histological features.: Insulin resistance, serum uric acid, metabolic syndrome, alanine aminotransferase and serum total cholesterol are a clue to pathogenesis and candidate targets for treatment. Hepatol Res 2016;46(11):1074-1087.

22. Fujii H, Imajo K, Yoneda M, Nakahara T, Hyogo H, Takahashi H, et al; Japan Study Group of Nonalcoholic Fatty Liver Disease. HOMA-IR: An independent predictor of advanced liver fibrosis in nondiabetic non-alcoholic fatty liver disease. J Gastroenterol Hepatol 2019;34(8):1390-1395.

23. Younossi Z, Anstee QM, Marietti M, Hardy T, Henry L, Eslam M, et al. Global burden of NAFLD and NASH: trends, predictions, risk factors and prevention. Nat Rev Gastroenterol Hepatol 2018;15(1):11-20.

24. Younossi ZM, Koenig AB, Abdelatif D, Fazel Y, Henry L, Wymer M. Global 
epidemiology of nonalcoholic fatty liver disease-Meta-analytic assessment of prevalence, incidence, and outcomes. Hepatology 2016;64(1):73-84.

25. Labenz C, Huber Y, Kalliga E, Nagel M, Ruckes C, Straub BK, et al. Predictors of advanced fibrosis in non-cirrhotic non-alcoholic fatty liver disease in Germany. Aliment Pharmacol Ther 2018;48(10):1109-1116.

26. Ulasoglu C, Enc FY, Kaya E, Yilmaz Y. Characterization of Patients with Biopsy-Proven Non-Alcoholic Fatty Liver Disease and Normal Aminotransferase Levels. J Gastrointestin Liver Dis 2019;28(4):427-431.

27. Alkayyali T, Qutranji L, Kaya E, Bakir A, Yilmaz Y. Clinical utility of noninvasive scores in assessing advanced hepatic fibrosis in patients with type 2 diabetes mellitus: a study in biopsy-proven non-alcoholic fatty liver disease. Acta Diabetol 2020;57(5):613-618.

28. Pattnaik K, Bhuyan P, Singh A, Singh SP, Nath P, Kar S, et al. Biopsy Proven Fibrosis in Non-Alcoholic Fatty Liver Disease: An Analysis of Risk Factors. J Clin Exp Hepatol 2018;8(4):367-374.

29. McPherson S, Hardy T, Henderson E, Burt AD, Day CP, Anstee QM. Evidence of NAFLD progression from steatosis to fibrosing-steatohepatitis using paired biopsies: implications for prognosis and clinical management. J Hepatol 2015;62(5):1148-1155.
30. Hossain IA, Rahman Shah MM, Rahman MK, Ali L. Gamma glutamyl transferase is an independent determinant for the association of insulin resistance with nonalcoholic fatty liver disease in Bangladeshi adults: Association of GGT and HOMA-IR with NAFLD. Diabetes Metab Syndr 2016;10(1 Suppl 1):S25-29.

31. Kim D, Kim W, Joo SK, Bae JM, Kim JH, Ahmed A. Subclinical Hypothyroidism and Low-Normal Thyroid Function Are Associated With Nonalcoholic Steatohepatitis and Fibrosis. Clin Gastroenterol Hepatol 2018;16(1):123-131.e1.

32. Slack A, Yeoman A, Wendon J. Renal dysfunction in chronic liver disease. Crit Care 2010;14(2):214.

33. Franko A, Merkel D, Kovarova M, Hoene M, Jaghutriz BA, Heni M, et al. Dissociation of Fatty Liver and Insulin Resistance in I148M PNPLA3 Carriers: Differences in Diacylglycerol (DAG) FA18:1 Lipid Species as a Possible Explanation. Nutrients 2018;10(9):1314.

34. Barata L, Feitosa MF, Bielak LF, Halligan B, Baldridge AS, Guo X, et al. Insulin Resistance Exacerbates Genetic Predisposition to Nonalcoholic Fatty Liver Disease in Individuals Without Diabetes. Hepatol Commun 2019;3(7):894-907. 